\title{
Contractile properties of the abductor digiti minimi muscle in paramyotonia congenita ${ }^{1}$
}

\author{
DAVID BURKE, NEVELL F. SKUSE, AND A. KEITH LETHLEAN \\ From the Unit of Clinical Neurophysiology, Division of Neurology, \\ Prince Henry Hospital, Little Bay, N.S.W. 2036, Australia
}

SYNOPSIS In two subjects with paramyotonia congenita the isometric torque generated by the $\vec{\circ}$ abductor digiti minimi and the surface EMG recorded over ADM decreased during prolonged or repetitive contractions, whether these were voluntarily or electrically induced. Isometric twitch times did not alter significantly during this muscle fatigue. Cooling greatly accelerated the fatiguing process. It is suggested that this local muscle weakness is due to a progressive decrease in excitability of the muscle cell membrane.

Muscular weakness is now recognized to play a major role in the symptomatology of paramyotonia congenita. Episodes of generalized paresis similar to hyperkalaemic periodic paralysis, or, rarely, hypokalaemic periodic paralysis, are well documented in some families with otherwise classical paramyotonia. For these reasons, the right of paramyotonia congenita to exist as a nosological entity distinct on the one hand from myotonia congenita and on the other from the periodic paralyses has been debated. A wellrecognized but less well-studied form of weakness in paramyotonia is that which develops in the exercised muscle and accompanies but outlasts the myotonia.

This paper analyses the properties of the abductor digiti minimi muscle (ADM) in two subjects with paramyotonia congenita to elucidate aspects of this local form of weakness that develops with muscle use. Features of myotonia will be analysed in a subsequent paper (Burke et al., 1974b).

\section{METHODS}

Two brothers, aged 24 and 19 years, with paramyotonia congenita were studied. The subjects presented with the classical clinical syndrome and a family history of a dominantly inherited condition which

1 Some of these findings were reported to the 1974 meeting of the Australian Association of Neurologists. could be traced back for five generations. Fugil clinical details are to be reported elsewhere. The elder $\mathrm{O}$ subject was studied in great detail in 13 separateexperimental sessions at weekly intervals. The data obtained were thus verified by repetition in the sange? subject, and the essential conclusions were then con firmed in the younger subject. These experimenti $\overrightarrow{0}$ sessions provided data for this report and another $f$ dealing with myotonia.

The mechanical and electrical properties of $\mathrm{ADM}$ were studied under isometric conditions using the techniques described in detail by Burke et al. (1974a). The torque produced by voluntary and electrically- $\bar{D}$ induced isometric contractions was recorded to- $\stackrel{2}{\Rightarrow}$ gether with the surface electromyogram (EMG) of $\frac{0}{3}$ ADM. In one experimental session the contractile properties of triceps surae were studied under isometric conditions. The results to be presented are those only of ADM but, since essentially similar data were obtained in triceps surae, it is reasonable to apply the conclusions more generally.

Experiments were carried out in a warm airconditioned laboratory. Skin temperature over ADM 0 was recorded routinely using an Ellab electronic thermometer, and was maintained at $34-35^{\circ} \mathrm{C}$ 윽 except during studies of the effects of cooling.

\section{RESULTS}

Muscle fatigue developed progressively through- $\stackrel{N}{N}$ out an experimental session whether contraction $\mathrm{W}$ was voluntary or electrically-induced. Fatigue ${ }^{\sigma}$ involved both maximal torque output and EMG, 
the peak to peak amplitude of the interference pattern decreasing to less than $150 \mu \mathrm{V}$ at the end of a prolonged experiment. After complete muscle fatigue, clinical recovery occurred slowly only after rest for one to two hours. This factor
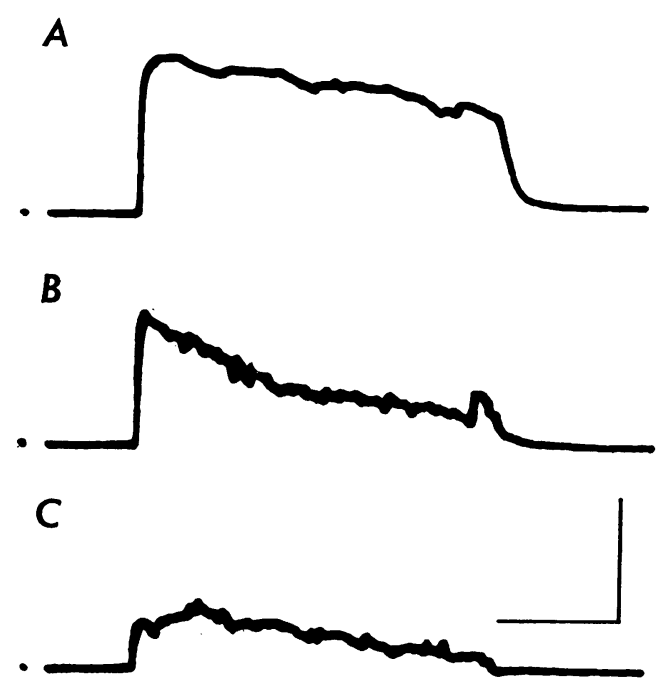

FIG. 1. Maintained voluntary contraction. Torque produced by three successive voluntary contractions, for A $10 \mathrm{~s}, \mathrm{~B} 30 \mathrm{~s}$, and C $30 \mathrm{~s}$. Muscle strength fatigues as duration increases, and recovery after cessation of contraction is incomplete. Calibrations: horizontal$3 \mathrm{~s}$ for $\mathrm{A} ; 10 \mathrm{~s}$ for $\mathrm{B}$ and $\mathrm{C}$; vertical-93.2 $\times 10^{-3} \mathrm{Nm}$ for $\mathrm{A}, \mathrm{B}$, and $\mathrm{C}$.

produced a variability which explains the large number of experimental sessions necessary to validate the experimental data completely.

MAINTAINED CONTRACTION The torque produced by maximal voluntary contraction of ADM varied from experiment to experiment depending on the subject's previous activities. The maximal recorded torque was $125.8 \times 10^{-3}$ Newtonmetres $(\mathrm{Nm})$. With sustained contraction, torque was usually well maintained for the initial 2-5 s but decreased by $30-40 \%$ after $5-10 \mathrm{~s}$ (Fig. 1A) and by $50-70 \%$ after $30 \mathrm{~s}$ (Fig. 1B). The EMG interference pattern declined along an asymptotic course, reaching $300 \mu \mathrm{V}$ within $10-15 \mathrm{~s}$, and then maintaining a relatively constant level with only slight further fall over the final $15 \mathrm{~s}$. After rest for $30 \mathrm{~s}$ recovery occurred but this was incomplete and repeated contractions induced fatigue over a similar time course starting from lower levels (Fig. 1C). Regularly repeated brief contractions appeared to be just as fatiguing as a prolonged contraction maintained for a similar duration. By contrast, in three normal subjects, maximal voluntary torque averaged $115.1 \times 10^{-3}$ $\mathrm{Nm}$, and fatigued by $23-31 \%$ after $30 \mathrm{~s}$ (six observations). This fatigue was not accom-

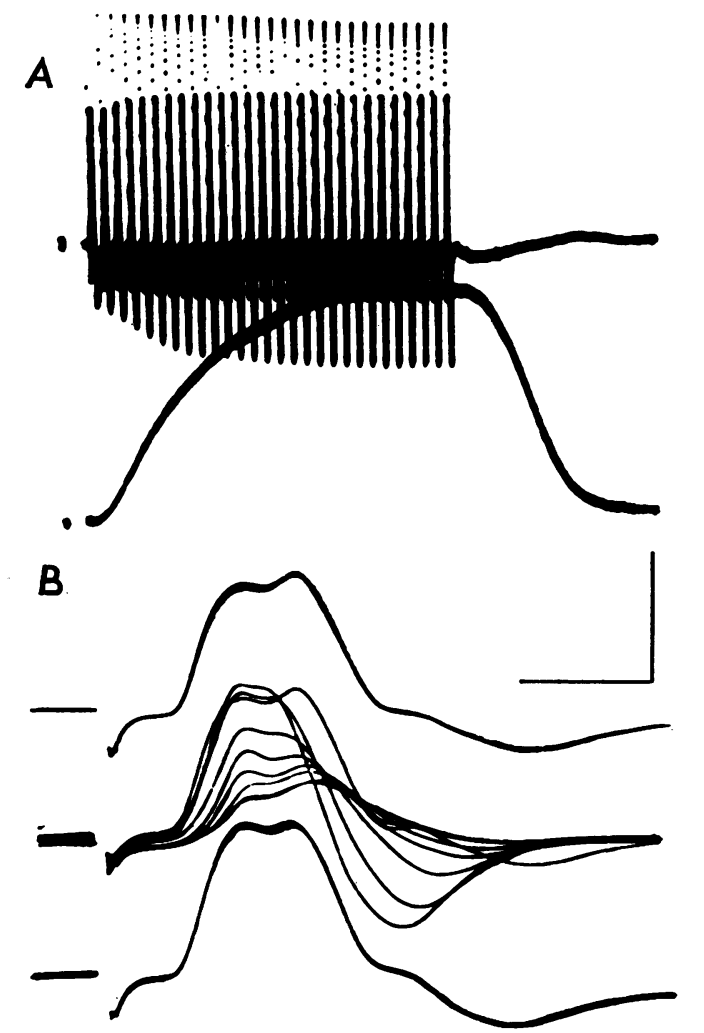

FIG. 2. Evoked MAP in response to tetanization at $50 \mathrm{~Hz}$. A: the MAP evoked by the initial stimuli increases in amplitude as tetanic torque rises. B: upper trace consists of three superimposed control responses before tetanus and the lower trace three control responses a few seconds after cessation of tetanus. In the middle trace the initial response to a tetanic train and then one response every few seconds throughout a $10 \mathrm{~s}$ tetanus are superimposed. The second MAP is of larger amplitude but shorter duration than in the control traces but thereafter the MAP decrements. Calibrations: horizontal-0.2 s for A; $4 \mathrm{~ms}$ for $\mathrm{B}$; vertical $-6 \mathrm{mV}$ and $28 \times 10^{-3} \mathrm{Nm}$ for $\mathrm{A} ; 3 \mathrm{mV}$ for $\mathrm{B}$. 
panied by a visible decrease of the EMG interference pattern, recovery was rapid and complete within $15 \mathrm{~s}$, and repeated contraction evoked similar changes.

The ulnar nerve at the wrist was tetanized at $50 \mathrm{~Hz}$ using supramaximal voltage levels after blocking the nerve at the elbow with lignocaine $2 \%$. The greatest tetanic torque recorded was $44.3 \times 10^{-3} \mathrm{Nm}$. Tetanization proved as effective a stimulus for muscle fatigue as voluntary contraction. In a series of three brief tetanic contractions, peak torque in the first contraction was $41.9 \times 10^{-3} \mathrm{Nm}$ decreasing to $21 \times 10^{-3} \mathrm{Nm}$ after $5 \mathrm{~s}$. In the second contraction peak torque was $33.6 \times 10^{-3} \mathrm{Nm}$ decreasing to $16.8 \times 10^{-3}$ $\mathrm{Nm}$ after $5 \mathrm{~s}$, and in the third, peak torque was $25.2 \times 10^{-3} \mathrm{Nm}$ decreasing to $14.9 \times 10^{-3} \mathrm{Nm}$ after $5 \mathrm{~s}$. Thus, although recovery occurred on cessation of stimulation, this was incomplete. Profound changes in the evoked muscle action potential (MAP) resulted from tetanization. In response to the first few stimuli the amplitude of the negative phase of the MAP increased but this was accompanied by a decrease in the duration of the potential so that there was little overall change in the area of the negative phase (Fig. 2). Thereafter, the amplitude of the negative phase progressively decreased, the degree of decrement depending on the duration of the stimulus train (Fig. 2B). Immediately after cessation of the stimulus train the MAP regained control amplitudes. Tetanization at $25 \mathrm{~Hz}$ produced a similar decrement of the MAP but a more prolonged stimulus train was required. Frequencies below $25 \mathrm{~Hz}$ did not produce MAP decrement.

Prolonged tetanization at $50 \mathrm{~Hz}$ produced a progressive fall in tetanic torque by an average of $59 \%$ (range $50-79 \%$ ) after $30 \mathrm{~s}$ (four observations). Tetanization in a slightly fatigued muscle was at least as effective in inducing these changes (Fig. 3A, B). Progressive decrease in amplitude of the evoked MAP accompanied the fatigue, the amplitude of the negative phase decreasing to $16 \%$ after $30 \mathrm{~s}$, but recovering on cessation of tetanization such that single stimuli evoked a MAP of control amplitude (Fig. 3C). By contrast, in three normal subjects, torque fatigued by $31 \%$ after tetanization for $30 \mathrm{~s}$, and this was not accompanied by decrement of the MAP (Burke et al., 1974a, Fig. 3).

Edrophonium $10 \mathrm{mg}$ was administered intra-
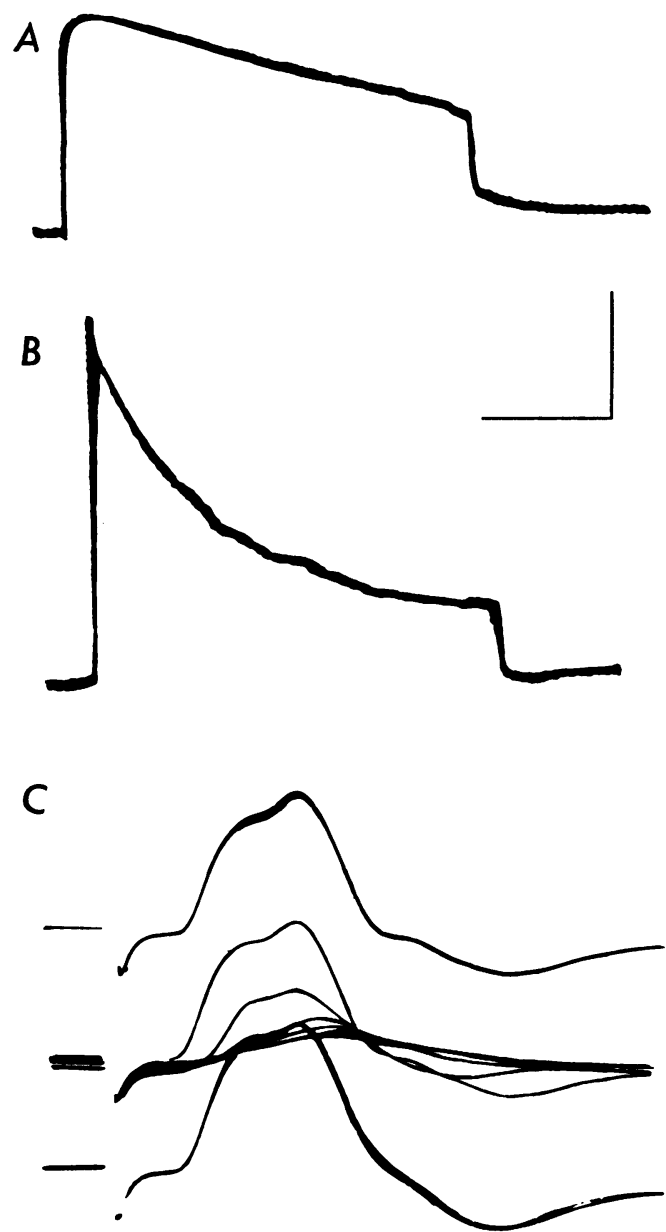

FIG. 3. Tetanization at $50 \mathrm{~Hz}$ for $30 \mathrm{~s}$. A and B: tetanic torque decreases in a relatively unfatigued muscle (A) by $50 \%$ in $30 \mathrm{~s}$ and in a relatively fatigued muscle (B) by $79 \%$ in $30 \mathrm{~s}$. C: the evoked MAP is shown as for Fig. $2 B$, one response being recorded every $5 \mathrm{~s}$ throughout the tetanic train in the middle trace. MAP amplitude decrements to less than $16 \%$ of the control amplitude. Calibrations: horizontal-10 s for $\mathrm{A}$ and $\mathrm{B} ; 4 \mathrm{~ms}$ for $\mathrm{C}$; vertical $-25 \times 10^{-3} \mathrm{Nm}$ for A; $7.5 \times 10^{-3} \mathrm{Nm}$ for B; $3 \mathrm{mV}$ for $\mathrm{C}$.

venously on two occasions. This did not affect established fatigue and did not alter the response to tetanic stimulation.

TWITCH CONTRACTION The twitch torque produced by supramaximal stimulation of the ulnar nerve at the wrist varied with the state of muscle 
fatigue. In rested muscle twitch torque ranged from $2.8 \times 10^{-3} \mathrm{Nm}$ to $6.3 \times 10^{-3} \mathrm{Nm}$ but with induced muscle fatigue this decreased to between $0.7 \times 10^{-3} \mathrm{Nm}$ and $1.3 \times 10^{-3} \mathrm{Nm}$. The decrease in twitch torque was accompanied by a decrease in MAP amplitude. Provided that limb temperature was maintained, twitch times did not alter significantly during muscle fatigue (Fig. 4). In the elder subject, contraction times varied between $63 \mathrm{~ms}$ and $70 \mathrm{~ms}$ and half-relaxation times between $68 \mathrm{~ms}$ and $80 \mathrm{~ms}$ in different experiments. The values for the younger subject were $70 \mathrm{~ms}$ and $78 \mathrm{~ms}$ respectively. These values fall within the normal range for ADM (Burke et al., 1974a).

Despite the fatigue induced in both torque and EMG during a tetanic contraction, post-tetanic potentiation of twitch torque by $25-33 \%$ could still be recorded immediately after cessation of tetanus. Repetitive stimulation at $3 \mathrm{~Hz}$ for $90 \mathrm{~s}$ induced the positive staircase phenomenon, the increase in twitch torque in the unfatigued state being $18 \%$. Both the post-tetanic potentiation and positive staircase phenomenon were not accompanied by a significant alteration in the evoked MAP, and such behaviour represents a normal response of the contractile mechanism.

Single motor unit contractions were elicited by stimulation at the wrist and at the motor point using threshold stimulus levels (Burke et al., 1974a). Six threshold twitches were averaged. Twitch torque of each unit was less than $4.66 \times 10^{-5} \mathrm{Nm}$, and the contraction times were $42,51,53,58,65$, and $68 \mathrm{~ms}$ (Fig. 5). It was additionally noted that even when ADM had been fatigued as completely as possible the motor point remained the point of lowest threshold for direct percutaneous stimulation of the muscle.

EFFECT OF COOLING The right hand was cooled in four experiments in the two subjects using ice packs. Temperature of the skin overlying ADM was monitored and was shown to decrease from $34-35^{\circ} \mathrm{C}$ to between $21^{\circ} \mathrm{C}$ and $28^{\circ} \mathrm{C}$ in different experiments. Cooling greatly accentuated the fatigue produced by voluntary or electrically induced contraction, both torque and EMG being affected in parallel until ultimately a stage just short of complete flaccid paralysis was reached. During cooling, voluntary contraction and tetanization produced a similar decline in
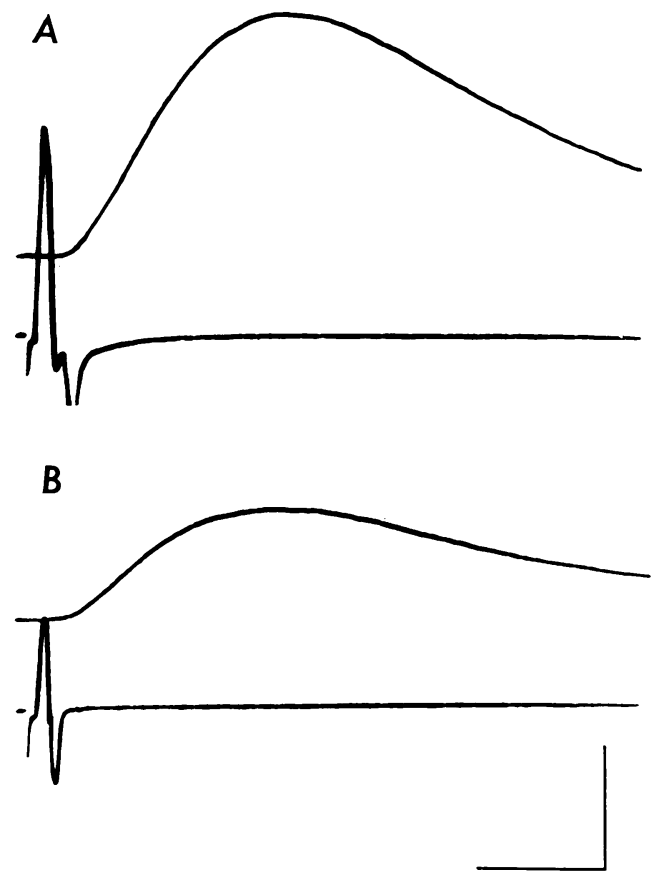

FIG. 4. Twitch contraction of ADM. A: in a relatively unfatigued muscle. Twitch torque $4.66 \times 10^{-3}$ $\mathrm{Nm}$. B: during the fatiguing process. Twitch torque $2 \cdot 1 \times 10^{-3} \mathrm{Nm}$. MAP amplitude has also fatigued but there is no significant change in twitch times. Calibrations: vertical $2.33 \times 10^{-3} \mathrm{Nm}$, and $3 \mathrm{mV}$; horizontal $40 \mathrm{~ms}$.

torque output and a similar decrement in EMG response as before cooling but these processes appeared accelerated and recovery was even less complete. On rewarming, fatigue persisted so that the muscle had not regained power by the conclusion of the experiments.

Twitch torque and the evoked MAP decreased

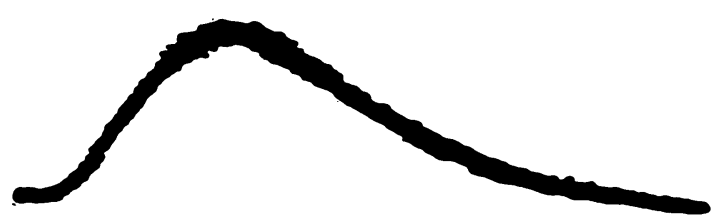

FIG. 5. Threshold motor unit contraction. Threshold stimuli were delivered at $2 \mathrm{~Hz}$ to the ulnar nerve at the wrist during arterial occlusion. Two hundred consecutive contractions have been averaged. Duration of trace-250 ms. Contraction time-58 ms. 
in parallel with the decrease in voluntary and tetanic torque. The time course of the twitch contraction increased dramatically, the half relaxation time being more profoundly affected than the contraction time. Details of the changes in twitch times will be presented in the companion paper analysing myotonia.

\section{DISCUSSION}

Weakness and stiffness are the major symptoms in the non-dystrophic forms of myotonia, and these symptoms may not be adequately differentiated by patient or physician. A close analysis of these symptoms in paramyotonia congenita suggests that the local paresis is flaccid and therefore cannot be dependent on the presence of myotonia. As demonstrated by this study, the local paresis may be analysed as a phenomenon independent of myotonia.

In paramyotonia, muscle power and other contractile properties appear initially within normal limits. The essential defect appears to be one of excessive fatiguability with repeated use. Muscle fatigue is a complex process. One factor could be the motor unit composition of ADM, which in normal man contains what are probably fast twitch fatigue-sensitive and fatigue-resistant motor units (Burke et al., 1974a). Conceivably, muscle fatigue in paramyotonia could be related to the presence of an abnormally large percentage of fast twitch fatigue-sensitive units. Certainly the contraction times of the six threshold units fall in the faster half of the normal range for ADM, but, since EMG decrement always accompanied muscle fatigue, such fatigue cannot be attributed completely to an abnormally large population of essentially normal motor units.

The EMG changes suggest that the fatiguing process cannot be attributed wholly to a primary defect of the contractile mechanism within the muscle cell, but probably arises from either a failure of neuromuscular transmission or a muscle cell membrane phenomenon. The pattern of EMG decrement is unlike that seen in established transmission defects such as the myasthenic syndromes, and anticholinesterase medication did not affect the process. Perhaps the most satisfactory explanation is that the muscle fatigue is a membrane phenomenon arising from progressive changes in excitability of the muscle cell membrane.

The effects of repetitive electrical stimulation on the evoked MAP have been studied by other authors, mainly in European literature. At normal temperatures, decrement has been reported to occur at $10 \mathrm{~Hz}$ (Garcin et al., 1966) and at $20 \mathrm{~Hz}$ (Isch et al., 1968), but not at $3 \mathrm{~Hz}$ and $5 \mathrm{~Hz}$ (Isch et al., 1968). No decrement was found at $10 \mathrm{~Hz}$ (Sigwald et al., 1971), at $20 \mathrm{~Hz}$ (Woolsey et al., 1969) and at $25 \mathrm{~Hz}$ (Ricker and Meinck, 1972), although when the muscle was cooled profound decrement was recorded with $25 \mathrm{~Hz}$ stimulation (Ricker and Meinck, 1972). The discrepancies between these reports probably represent variations in severity of the disorder in the cases studied. Together with the present experiments, these studies emphasize the apparent normality of the initial response to stimulation and the necessary role of repeated stimulation to bring out the muscular defect. Cooling may produce conditions under which this defect may be recognized, but would appear to play only an aggravating role.

The myotonia of paramyotonia congenita is commonly 'paradoxical', in that it increases with repeated contraction of muscle. Thus, the two phenomena which interfere with the volitional control of muscular function are brought out by repetitive or prolonged use. These phenomena may take place at room temperature and may cause significant disability even in warm environments, and, although cooling accentuates the defect, it does not appear to introduce any new factor not seen at normal or elevated temperatures. Since aggravation by cooling is a phenomenon not restricted to paramyotonia and since the stimulus necessary to demonstrate the unique muscular defects of paramyotonia is repeated muscle contraction, it would seem wise to de-emphasize the effects of cooling and to redefine paramyotonia in terms of paradoxical myotonia and muscle fatigue.

\section{SUMMARY}

The contractile properties of the abductor digiti minimi muscle (ADM) have been studied under isometric circumstances in two subjects with paramyotonia congenita to analyse the local weakness which develops in exercised muscles. 
Torque and EMG produced by muscle contraction progressively fatigued throughout an experiment whether contraction was voluntary or electrically-induced. These changes took place at room temperature and were accentuated by muscle cooling. Maximal torque decreased by $50-70 \%$ during maintained voluntary contraction for $30 \mathrm{~s}$ and by $50-79 \%$ during tetanization at $50 \mathrm{~Hz}$ for $30 \mathrm{~s}$. EMG decrement accompanied the muscle fatigue, the amplitude of the evoked muscle action potential (MAP) decreasing by more than $80 \%$ after tetanization at $50 \mathrm{~Hz}$ for $30 \mathrm{~s}$. Similar EMG decrement occurred with repetitive stimulation at $25 \mathrm{~Hz}$, but not at frequencies below this.

Total muscle twitch times were within normal limits in unfatigued muscle, and did not change appreciably as muscle fatigue developed, even though twitch torque and MAP amplitude decreased. On repetitive activation at $3 \mathrm{~Hz}$ the positive staircase phenomenon was demonstrable and after tetanization post-tetanic potentiation of twitch torque was recorded, much as in normal subjects.

These data suggest that in paramyotonia congenita muscle fatigue is a membrane phenomenon, presumably due to a progressive decrease in excitability of the muscle cell membrane.

The authors are grateful to Mr Peter van Megen and A.C.I. Ltd without whose generous assistance these studies could not have been performed, and to Professor J. W. Lance for advice in the preparation of this report. Illustrations were photographed by the Department of Medical Illustration, University of New South Wales.

\section{REFERENCES}

Burke, D., Skuse, N. F., and Lethlean, A. K. (1974a). Isometric contraction of the abductor digiti minimi muscle in man. Journal of Neurology, Neurosurgery, and Psychiatry. (In press.)

Burke, D., Skuse, N. F., and Lethlean, A. K. (1974b). An analysis of myotonia in paramyotonia congenita. Journal of Neurology, Neurosurgery, and Psychiatry, 37, 900-906.

Garcin, R., Legrain, M., Rondot, P., and Fardeau, M. (1966). Etude clinique et métabolique d'une observation de paramyotonie congénitale d'Eulenburg. Documents ultrastructuraux concernant la biopsie musculaire. Revue Neurologique, 115, 295-311.

Isch, F., Isch-Treussard, C., Jésel, M., and Warter, J. M. (1968). Étude électromyographique de deux cas de paramyotonie congénitale de von Eulenburg. Revue Neurologique, 118, 536-542.

Ricker, K., and Meinck, H.-M. (1972). Paramyotonia congenita (Eulenburg). Neurophysiologic studies of a case. Zeitschrift für Neurologie, 203, 13-22.

Sigwald, J., Rondot, P., Fardeau, M., and Godet-Guillain, J. (1971). Paramyotonie congénitale d'Eulenburg. Étude clinique, biologique, histologique et ultrastructurale d'une nouvelle observation familiale. Revue Neurologique, 124, 439-450.

Woolsey, R. M., Nelson, J. S., and Rossini, A. A. (1969). Paramyotonia and progressive neurogenic atrophy. Neurology (Minneap.), 19, 909-914. 